\title{
Cell adhesion molecules in Drosophila synapse development and function
}

\author{
SUN MingKuan* \& XIE Wei* \\ Key Laboratory of DGHD, MOE, Institute of Life Sciences, Southeast University, Nanjing 210096, China
}

Received July 7, 2011; accepted September 15, 2011

\begin{abstract}
Synapse is a highly specialized inter-cellular structure between neurons or between a neuron and its target cell that mediates cell-cell communications. Ample results indicate that synaptic adhesion molecules are critically important in modulating the complexity and specificity of the synapse. And disruption of adhesive properties of synapses may lead to neurodevelopmental or neurodegenerative diseases. In this review, we will use the Drosophila NMJ as a model system for glutamatergic synapses to discuss the structure and function of homophilic and heterophilic synaptic adhesion molecules with special focus on recent findings in neurexins and neuroligins in Drosophila.
\end{abstract}

Drosophila, cell adhesion molecules, synapse, neuromuscular junction, neurexin, neuroligin

Citation: $\quad$ Sun M K, Xie W. Cell adhesion molecules in Drosophila synapse development and function. Sci China Life Sci, 2012, 55: 20-26, doi: 10.1007/ s11427-012-4273-3

Synapse is a specialized cell adhesion structure between a neuron and its target cell or another neuron. It is involved in neural information processing and storage. Synapse formation is a multi-step process that includes target recognition that requires cell adhesion molecules to initiate cell-cell contact, and subsequent recruitment of proteins and organelles to form the presynaptic and postsynaptic compartments. Many cell adhesion molecules (CAMs) are expressed at the synapse. These are membrane-anchored molecules, consisting of three domains: an intracellular domain that can directly interact with intracellular proteins to mediate signaling processes, a transmembrane domain, and an extracellular domain that interacts either with another molecule of the same CAM protein (homophilic binding) or with different CAM or extracellular matrix proteins (heterophilic binding). Disruption of adhesive properties of synapses may lead to neurodevelopmental or neurodegenerative diseases, such as autism and schizophrenia [1].

*Corresponding author (email: sunmk@ seu.edu.cn; wei.xie@ seu.edu.cn)
Drosophila melanogaster has been widely used as a model system to address fundamental questions related to neuroscience not only because it has a relatively simpler genome and nervous system, but also because many molecular mechanisms underlying neuronal development that operate in mammals, including those involved in synaptogenesis, are highly conserved in this organism. In addition, the fruit fly has a well-characterized repertoire of molecular genetic tools and a relatively short life span [2], thus providing an invaluable model system for genetic dissections of the nervous system on a large scale. In particular, the Drosophila neuromuscular junction (NMJ), an asymmetric chemical synapse formed between motor neurons and muscle cells, is an excellent model system for investigating the fundamental mechanisms governing synaptic development and function. The advantageous features of the Drosophila NMJ include its structural accessibility, stereotypic feature and amenability to genetic manipulations, electrophysiological and microscopic analyses [3-7].

The Drosophila NMJ is a glutamatergic synapse. Many 
of its properties, including the molecular composition, are similar to those of excitatory synapses of mammalian CNS, therefore, the Drosophila NMJ is considered as a convenient and useful model for elucidating the mechanisms underlying glutamatergic synapses [8,9]. Indeed, the NMJ has been a site of extensive investigations in the context of synapse formation, synaptic transmission and plasticity, and synaptic degeneration.

Here, we summarize recent findings on the roles of selected synaptic adhesion molecules in synapse development, with special emphasis on the Drosophila neurexin-neuroligin complex. Our key focus is to highlight the Drosophila Neurexin-neuroligin complex principles that govern the molecular basis of synapse formation and function. We conclude by providing a hypothetical model for neurexin-neuroligin signaling at the NMJ.

\section{Homophilic CAMs}

The identified homophilic CAMs at the Drosophila NMJ include Capricious, Connectin, Fasciclin II, Fasciclin III, Neuroglian, N-Cadherin and Dscam.

\subsection{Capricious}

Capricious (Caps) is a transmembrane protein with 14 leucine-rich repeats (LRRs). It is expressed presynaptically in the anterior corner cell (aCC), RP2, U, and RP5 motor neurons, and postsynaptically in muscles $1,2,9,10$, and 12 [10]. The level of Caps is regulated by the transcription factor Kruppel. Caps is necessary for proper defasciculation of SNb axons [11]. Loss-of-function or ectopic expression of the caps gene alters the target specificity of muscle 12 motorneurons [10,12] (Table 1). In muscle 12 cells, Caps is localized at the tips of myopodia before the arrival of motoneuronal growth cones. In caps mutants, there are fewer contacts between myopodia of M12 and the presynaptic growth cones during the initial neuromuscular interaction. In addition, the nascent synaptic sites of M12 are also reduced in the caps mutants. These results indicate that Caps is required for target recognition at the tips of myopodia [13].

\subsection{Connectin}

Connectin (Con) is a cell surface protein with 10 LRRs, which may mediate homophilic interaction in vitro [14]. The expression of Con is first observed in one to three myoblasts on the lateral side of the body wall at late stage 11 to early stage 12 . Con is expressed on the surface of eight muscles, their innervating motoneurons and several surrounding glial cells. The connectin mutants or transgenic flies with ectopic expression do not show any significant neuromuscular defects, therefore, the role of this CAM remains elusive [15] (Table 1).

\subsection{Fasciclin II}

Fasciclin II (Fas II) is a homophilic interaction protein $[16,17]$ that has multiple functions in the Drosophila nervous system. The fact that the overall structural features of Fas II are remarkably similar to the vertebrate neuronal cell adhesion molecule (NCAM) suggests that it may represent

Table 1 Cell adhesion molecules at the Drosophila NMJ

\begin{tabular}{|c|c|c|c|}
\hline Genes & Expression patterns & Functions & References \\
\hline \multirow[t]{2}{*}{ Capricious } & Motor neurons aCC, RP2 U, RP5 & Defasciculation of SNb axons & [11] \\
\hline & muscle $1,2,910,12,1415,16,17$ and 30 & Target recognition & [12] \\
\hline Connectin & Muscle 5,8,18, 21-24 and innervate their motor neurons & Muscle pattern formation & [14] \\
\hline \multirow[t]{2}{*}{ FasciclinII } & All motor neurons & Presynaptic cell pattern formation & [26] \\
\hline & All muscles & Postsynaptic accumulation & \\
\hline FasciclinIII & RP3 motor neuron muscle $6 / 7$ & Target recognition & [31] \\
\hline Neuroglian & $\begin{array}{l}\text { All motor neurons } \\
\text { All muscles }\end{array}$ & Target recognition & {$[35]$} \\
\hline N-Cadherin & $\begin{array}{l}\text { All motor neurons } \\
\text { All muscles }\end{array}$ & Axonal pattern formation & {$[40]$} \\
\hline Dscam & $\begin{array}{l}\text { All motor neurons } \\
\text { All muscles }\end{array}$ & $\begin{array}{l}\text { Presynaptic cell pattern formation } \\
\text { Dendritic development }\end{array}$ & [41] \\
\hline \multirow[t]{2}{*}{ Neurexin IV } & $\begin{array}{l}\text { Midline neurons } \\
\text { Glia cells }\end{array}$ & Epithelial and axo-glial SJs & [47] \\
\hline & Epithelial Cells & Axon guidance & \\
\hline \multirow[t]{2}{*}{ Neurexin-1 } & CNS neurons & Synaptic assembly & {$[50]$} \\
\hline & Body Wall muscles & Learning and memory & {$[52]$} \\
\hline Neuroligin1 & Body Wall muscles & Synaptic growth & [54] \\
\hline Neuroligin2 & $\begin{array}{c}\text { CNS neurons } \\
\text { Motor neurons } \\
\text { Body Wall muscles } \\
\end{array}$ & $\begin{array}{l}\text { Postsynaptic differentiation } \\
\text { Synaptic growth } \\
\text { DGluRs recruiment }\end{array}$ & {$[56]$} \\
\hline
\end{tabular}


the fly ortholog of the mammalian NCAM. Fas II has been shown to be important for the development, maintenance, and plasticity of the NMJ.

Fas II is expressed by differentiating neuroblasts during early neurogenesis in the Drosophila embryo [18,19], where it is involved in the induction of downstream proneural genes, including achaete (ac) and atonal (ato) [20]. Fas II is expressed in all motoneuron axon pathways, including growth cones from early axonal outgrowth to the time of synapse formation [21]. It is also expressed at low levels in all muscle cells [22].

Overexpression of Fas II in presynaptic neurons results in fusion of motoneuron axons, whereas decreased expression of Fas II leads to a complete or partial defasciculation of motor axon pathways [23,24]. In aCC and RP2 pioneer axons, Fas II is necessary and sufficient for guiding follower axons and the establishment of presynaptic cell patterns [25]. At the NMJ, FasII is expressed at both presynaptic and postsynaptic site and is required for the accumulation of scaffolding protein Discs large (Dlg) and glutamate receptor subunits (GluRII A and GluRII B) [26]. In some muscle cells, transient expression of Fas II results in the formation of new ectopic functional synapses [22]. These results suggest that Fas II plays essential roles in pattern formation and postsynaptic specialization (Table 1).

Recent work has also shown that Fas II and Dlg function together to modulate activity-dependent synaptic development and that this role is regulated by activation of CaMKII $[27,28]$. In addition, axonal Fas II can interact homophilically with a glial isoform of Fas II and this interaction is critical for the glial cell migration and is regulated by Fzr/Cdh1[29].

\subsection{Fasciclin III}

Fasciclin III (Fas III) is a single transmembrane, homophilic immunoglobulin superfamily (Ig CAM) protein [30]. It plays an important role in cell adhesion, axon pathfinding and fasciculation $[31,32]$.

Fas III is expressed in muscle 6 and 7, in the axons of RP motoneurons, including RP3. Fas III mutants displayed defects of RP3 axons guidance, which axons incorrectly innervate their targets. RP3 mistarget the neighboring muscles misexpressing Fas III [31]. Cell-specific expression pattern of Fas III may provide the molecular basis for its target recognition function (Table 1).

\subsection{Neuroglian}

Neuroglian ( $\mathrm{Nrg})$ is a homophilic interaction protein that contains six Ig-like domains and $5 \mathrm{FN}$ type III domains. It is related to a number of vertebrate CAMs although most closely to the mouse L1. Alternative splicing of the $\mathrm{Nrg}$ gene generates 2 isoforms; the long form of Nrg that is expressed on the surface of specific CNS and PNS neurons, as well as in some PNS support cells, and the short form of $\mathrm{Nrg}$ that is expressed in glia and a variety of other non-neuronal tissues, including trachea, hindgut, salivary gland and muscle [33]. Together with Ank, Nrg mediates the neuron-glia interaction to contribute to the axonal and dendritic morphogenesis [34]. Loss of function of Nrg results in motoneuron axon misprojections and stalling close to the target postsynaptic muscle cell [35]. Nrg in sensory neurons is also necessary for the maintenance of sensory axon advance [36] (Table 1).

\section{$1.6 \quad$ N-Cadherin}

The Drosophila N-cadherin (N-Cad) is an evolutionarily conserved, classic type cadherin with a large, complex extracellular domain and a catenin-binding cytoplasmic domain. There are 12 isoforms of $\mathrm{N}-\mathrm{Cad}$ that share the same molecular architecture but have different sequences in their transmembrane and extracellular domains, which mediate homophilic interactions [37-39]. N-Cad regulates axonal pattern formation, presumably by regulating axonal fasciculation in the developing embryo [40].

\subsection{Dscam}

The Drosophila Dscam, a homologue of human Down syndrome cell adhesion molecule (DSCAM), is an immunoglobulin (Ig) superfamily protein. It participates in the presynaptic motor neuron pattern formation at the $\mathrm{NMJ}$ and is important for precise neuronal connections in the fly brain [41]. Dscam is also required for dendritic self-avoidance in all four classes of Drosophila dendritic arborization (da) neurons [42]. The dendrites of these neurons distinguish self and non-self through Dscam1 homophilic interactions [41]. Drosophila Dscam1 could generate as many as 19008 different ectodomains by alternative splicing of three exon clusters, with each encoding half or a complete variable immunoglobulin domain. This isoform diversity provides the molecular basis for establishing specific self-avoidance in neurons with complex dendritic arborization [43].

\section{Heterophilic CAMs}

\subsection{Neurexin IV}

Neurexin IV (NRX IV) is a transmembrane protein with a cytoplasmic domain homologous to glycophorin $\mathrm{C}$ and is shown to be more similar to the vertebrate Caspr protein [44]. NRX IV is localized to septate junctions (SJs) of epithelial and glial cells, and is required for the formation and function of septate junction and blood-nerve barrier [45], and the establishment of epithelial cell polarity [46]. NRX IV is also expressed in the medline neurons, where it interacts with Roundabout and plays a role in repulsive midline 
axon guidance and is found a novel interacting component of the Robo/Slit signaling pathway [47-49] (Table 1).

\subsection{Neurexin-1}

The Drosophila Neurexin-1 (DNRX) is a single transmembrane protein with six LamininA/ Neurexin/sex hormonebinding protein domains and three interspersed epithelium growth factor like sequences. The DNRX protein has an identical domain structural organization to mammalian a-neurexins [50-52]. DNRX is expressed throughout the development of the nervous system. During embryonic stages, strong expression is observed along the longitudinal tracts of the VNC and brain. In the adult brain, DNRX is expressed at high levels within the medulla, lobula, lobula plate, mushroom body and antenal lobe [52]. Within synaptic boutons, DNRX mostly localizes to the active zone (AZ, also called T-bar) of presynaptic terminals. However, DNRX is present both pre- and post-synaptically in embryo and third instar larvae stages [50]. dnrx loss of function causes reduced proliferation of synaptic boutons at glutamatergic neuromuscular junctions whereas overexpression of DNRX in neurons leads to synaptic overgrowth [51].

DNRX promotes presynaptic AZ specialization, neurotransmitter release, and postsynaptic glutamate receptor clusters [50]. Thus, dnrx null mutants display striking defects in synaptic ultrastructure, including the presence of detachment-between pre- and postsynaptic membranes, abnormally long AZs, increased number of $\mathrm{T}$ bars, and deficits in synaptic transmission [52]. DNRX is also required for synapse formation in the adult CNS and is important for associative learning [52]. Therefore, DNRX is critically involved in both synapse development and function [50-52].

\subsection{Neuroligins}

There are four neuroligin genes identified in the Drosophila melanogaster genome. Their encoded proteins (CG13772, CG34127, CG34139, and CG31146) share significant similarity in amino acid sequence and predicted structure with the vertebrate Neuroligins: a type I membrane protein with an extracellular domain consisting of mostly of a region homologous to acetylcholinesterases but lacking the esterase activity, a transmembrane domain, and a cytoplasmic domains with a PDZ binding motif. Phylogenetic analysis based on protein sequences indicates that the fly neuroligins and mammalian neuroligins share a common ancestor [53].

Of the four neuroligin genes, only the transcript of Drosophila neuroligin 1 (dnll) is specifically expressed in muscle tissues as judged from in situ hybridizations. DNL1 protein (CG31146) is also specifically found in postsynaptic muscle cells and accumulates at NMJs, in a site adjacent to PSDs. Moreover, DNL1 forms discrete clusters at the edge of postsynaptic receptor fields [54]. dnl2 (CG13772) mRNA is primarily detected in the brain and $\mathrm{VNC}$ in embryonic stage 14, which is consistent with the expression pattern of DNL2. High levels of DNL2 are also observed in the brain and VNC of third-instar larvae, where it colocalizes with DNRX. DNL2 expression in the CNS appears to be widespread and uniform with no preferential colocalization with any specific neurotransmitter or neuro-hormone. DNL2 is also detected in the abdominal muscles of third-instar larvae, where it is enriched at the NMJs [53]. The expression of the other two dnls (CG34127 and CG34139) is also prominent in the CNS and third instar larval NMJs (unpublished).

Ample results indicate that DNL1 is required for effective addition of synaptic boutons at developing NMJ terminals, and postsynaptic differentiation, including the accumulation of postsynaptic glutamate receptors, scaffold proteins, and subsynaptic membrane components. Because mutant animals of dnll showed overgrown glutamate receptor fields, at the same time, boutons that are positive for presynaptic markers frequently lacked postsynaptic receptor fields [54]. Meanwhile ectopic DNL1 expression triggers ectopic postsynaptic differentiation via its cytoplasmic domain.

DNL1 plays an important role in the regulation of synaptic function. In dnll mutant flies, eEJC amplitudes are reduced to approximately $50 \%$ of control levels at both low and high extracellular $\left[\mathrm{Ca}^{2+}\right]$, possibly due to less AZs formed. Moreover, eEJC decay kinetics is also prolonged in the dnll mutants, which might be in agreement with enlarged postsynaptic receptor fields. Overall amplitudes and frequencies of mEJCs are, however, not altered significantly from controls [54].

Null mutants of $d n l 2$ display reduced axonal branching and fewer synaptic boutons with an increase in the number of AZs per bouton, but a decrease in the thickness of subsynaptic reticulum (SSR) and in the length of postsynaptic densities [53]. dnl2 mutants also exhibit a decrease in the total glutamate receptor density and a shift in the subunit composition of glutamate receptors in favor of GluR IIA complexes. dnl2 mutants also show increased neurotransmitter release and altered kinetics of stimulus-evoked transmitter release [53].

\section{Trans-synaptic neurexin-neuroligin signaling at Drosophila NMJs}

Although CAMs have been shown to regulate synapse development and function, the exact underlying molecular mechanisms and in vivo significance remain unclear. It is known, however, that mutations in genes encoding several CAMs, such as neurexin and neuroligin, are linked to human neurological and mental disorders, including autism and schizophrenia [1]. In vitro studies suggest that the vertebrate neurexins and neuroligins play critical roles in the initial establishment of the synapse (reviewed in $[1,55]$ ), but in vivo results obtained from knockout animals suggest that 
these molecules are only important for the maturation or validation of the synapse. However, this conclusion is complicated by the existence of multiple genes and splice variants for both neurexins and neuroligins in mammals. In contrast, the Drosophila has relatively fewer members/splice variants of neurexin and neuroligins, providing some advantages to analyze the in vivo function and underlying mechanism [50-54].

DNRX localizes at the presynaptic membrane, where its intracellular domain interacts with $\mathrm{CAKI} / \mathrm{CMG}$, the Drosophila homolog of the vertebrate CASK, a member of the MAGUK scaffolding protein family. This interaction is important for synaptic vesicle trafficking [56]. dnrx mutants are reduced in the expression of Bruchpilot (BRP), a presynaptic protein known to be important for the structural integrity and function of synaptic AZs in Drosophila. BRP mutants exhibit deficits in T-bars, calcium channel clustering, and synaptic short-term plasticity [57,58]. Some of these deficits are also observed in dnrx mutants [57,58]. These results suggest that DNRX may regulate presynaptic assembly and functional maturation through interacting with BRP [59].

Interestingly, Drosophila Syd-1 (DSyd-1) has been identified to interact with BRP, and $d s y d-1$ mutants display smaller terminals with fewer release sites and reduced neurotransmitter release. In addition, the remaining AZs of the mutants are often large, ectopic, and abnormal in shape with electron-dense accumulations of BRP in boutons and axons [60]. These alterations are similar to those observed at $d n r x$ deficient NMJs [51]. Therefore, it is possible that DNRX functions in AZ assembly by forming BRP-DSyd-1-DNRX complexes (Figure 1). It has been proposed that DSyd-1 regulates effective nucleation of newly forming AZs via interacting with DLiprin- $\alpha$ [60].

In addition to presynaptic deficits, both $d n r x$ and $d s y d-$ 1mutants are also altered in postsynaptic specialization. Although DNRX localizes predominantly close to AZs, dnrx mutants show unexpected changes in the levels of glutamate receptor and DNL1 at the NMJs [50,51,54]. Similarly, dsyd-1 mutants also exhibit alterations in glutamate receptors at NMJs. These results suggest that DNRX and DSyd-1 are also important in the regulation of postsynaptic glutamate receptors, likely through trans-synaptic interaction with postsynaptic DNLs.

Interestingly, the postsynaptic deficits associated with $d n r x$ and $d s y d-1$ mutants are remarkably similar to those found in $d n l l$ and $d n l 2$ mutants. For example, loss of DSyd-1 or DNL2 leads to a dramatic increase in the amount

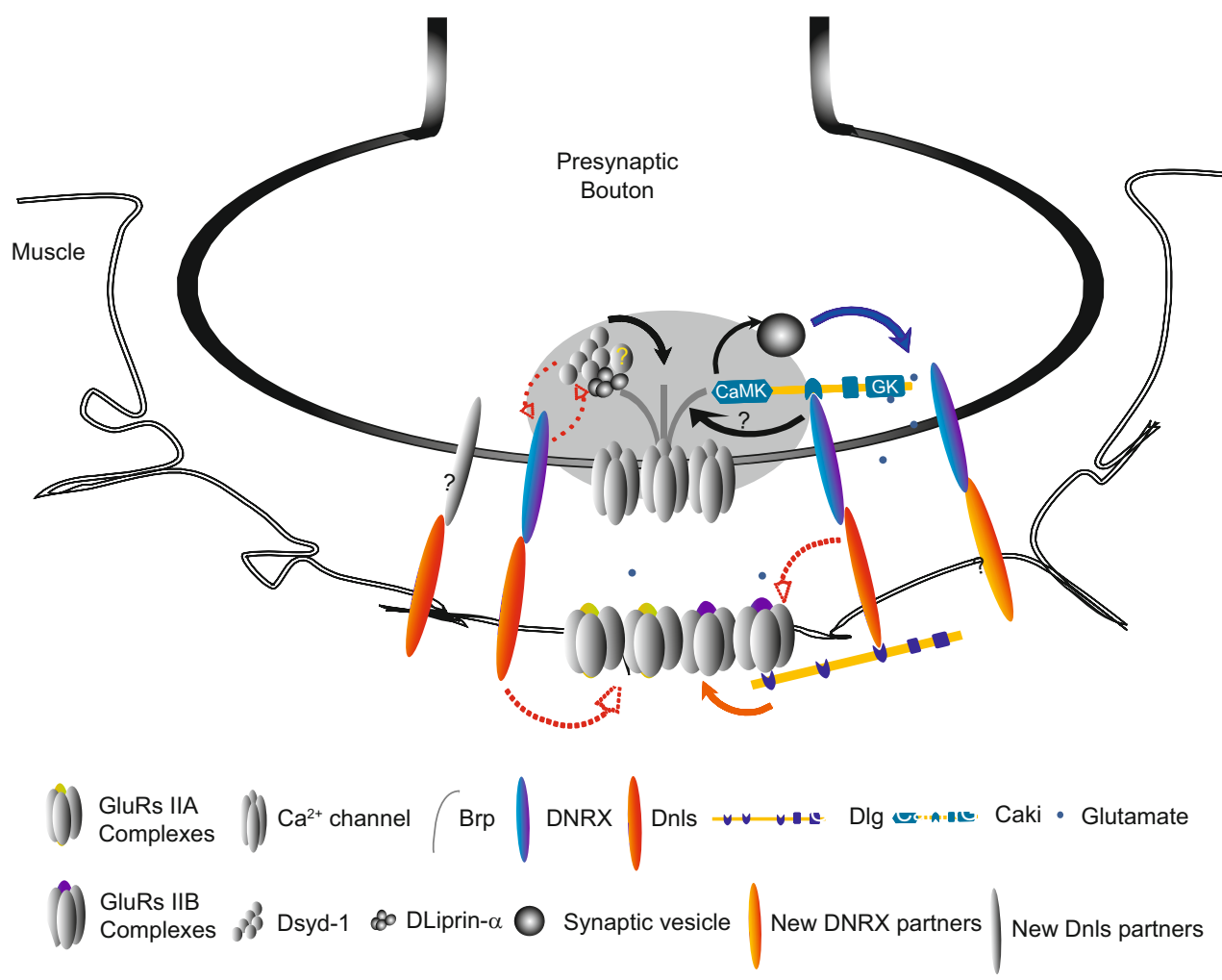

Figure 1 Model of neurexin-neuroligin mediated intercellular signaling. DNRX and DNL bind to each other to bridge the presynaptic and postsynaptic sites and strengthen signaling across the synapse. DNRX interacts with CASKI at the presynaptic site to regulate synaptic vesicle trafficking; DNRX also forms a protein complex with BRP and Dsyd-1 and this complex is important for the formation of active zone; DSyd-1 directly interacts with BRP at the AZ to regulate the localization and motility of Liprin- $\alpha$; DNRX is proposed as a direct substrate for DSyd-1. DNL can be regulated by DSyd-1 or other presynaptic proteins via trans-synaptic DNRX-DNL interaction, which in turn regulates postsynaptic receptor fields. Other potential DNRX and DNL interacting partners may also participate in this trans-synaptic signaling. The broken lines indicate links (direct or indirect) of unknown nature. 
of DGluRIIA and a decrease in DGluRIIB, resulting in a shift in the relative ratios of these receptors. These results are consistent with the idea that DNRX and Dsyd-1 regulate postsynaptic properties through DNRX-DNL trans-synaptic interaction. Both genetic and biochemical evidence supports a direct interaction of DNRX and DNL2 in Drosophila [53]. Exactly how DNL2 regulates GluRs remains unknown, but direct protein interactions with DLG may play an important role in this process $[51,53,60]$ (Figure 1 ).

Considering the overall phenotypes in mutant $d n r x$ [50-52] and $d n l[53,54]$, it is reasonable to conclude that DNRX and DNL regulate synaptic development and function at the Drosophila NMJ through DNRX-DNL trans-synaptic interaction. However, the fact that the $d n r x$ phenotype is weaker than the $d n l 1$ phenotype suggests that not all DNRX function is mediated by DLN1 [53]; indeed, $d n l 2$ null flies also display a significant reduction in the number of synaptic boutons. In addition, $d n r x / d n l 2$ double mutants exhibit more severe phenotypes than those observed in $d n r x$ or $d n l 2$ single mutants [53]. These results suggest that additional postsynaptic proteins may interact with and mediate the effect of DNRX. LRRTM2 has been recently identified as a synaptic cell-adhesion molecule that could act as a NRX receptor in mammals [61,62] and this may also occur in Drosophila. It is also possible that other partners than NRX exist for DNL. To identify these novel molecules and their specific roles in the regulation of synaptic development and function will be one of most important research topics and the fruit flies will continue to provide a useful system to investigate them.

We thank Drs. Jia ZhengPing and Zhou ZiKai for reading the manuscript and comments. This work was supported by the National Natural Science Foundation of China (Grant Nos. 31171041 and 31000486) and National Basic Research Program of China (Grant No. 2012CB517903).

1 Sudhof T C. Neuroligins and neurexins link synaptic function to cognitive disease. Nature, 2008, 455: 903-911

2 Adams M D, Celniker S E, Holt R A, et al. The genome sequence of Drosophila melanogaster. Science, 2000, 287: 2185-2195

3 Jan L Y, Jan Y N. Properties of the larval neuromuscular junction in Drosophila melanogaster. J Physiol, 1976, 262: 189-214

4 Keshishian H, Broadie K, Chiba A, et al. The Drosophila neuromuscular junction: a model system for studying synaptic development and function. Annu Rev Neurosci, 1996, 19: 545-575

5 Koh Y H, Gramates L S, Budnik V. Drosophila larval neuromuscular junction: molecular components and mechanisms underlying synaptic plasticity. Microsc Res Tech, 2000, 49: 14-25

6 Prokop A. Integrating bits and pieces: synapse structure and formation in Drosophila embryos. Cell Tissue Res, 1999, 297: 169-186

7 Richmond J E, Broadie K S. The synaptic vesicle cycle: exocytosis and endocytosis in Drosophila and C. elegans. Curr Opin Neurobiol, 2002, 12: 4997-507

8 Marrus S B, Portman S L, Allen M J, et al. Differential localization of glutamate receptor subunits at the Drosophila neuromuscular junction. J Neurosci, 2004, 24: 14067-1415

9 Schuster C M, Ultsch A, Schloss P, et al. Molecular cloning of an invertebrate glutamate receptor subunit expressed in Drosophila muscle. Science, 1991, 254: 112-114

10 Shishido E, Takeichi M, Nose A. Drosophila synapse formation: regulation by transmembrane protein with Leu-rich repeats, CAPRICIOUS. Science, 1998, 280: 2118-2121

11 Abrell S, Jackle H. Axon guidance of Drosophila SNb motoneurons depends on the cooperative action of muscular Kruppel and neuronal capricious activities. Mech Dev, 2001, 109: 3-12

12 Taniguchi H, Shishido E, Takeichi M, et al. Functional dissection of Drosophila capricious: its novel roles in neuronal pathfinding and selective synapse formation. J Neurobiol, 2000, 42: 104-116

13 Kohsaka H, Nose A. Target recognition at the tips of postsynaptic filopodia: accumulation and function of Capricious. Development, 2009, 136: 1127-1135

14 Nose A, Umeda T, Takeichi M. Neuromuscular target recognition by a homophilic interaction of connectin cell adhesion molecules in Drosophila. Development, 1997, 124: 1433-1441

15 Raghavan S, White R A. Connectin mediates adhesion in Drosophila. Neuron, 1997, 18: 873-880

16 Wright J W, Snyder M A, Schwinof K M, et al. A role for fasciclin II in the guidance of neuronal migration. Development, 1999, 126: 3217-3228

17 Grenningloh G, Rehm E J, Goodman C S. Genetic analysis of growth cone guidance in Drosophila: fasciclin II functions as a neuronal recognition molecule. Cell, 1991, 67: 45-57

18 Urbach R, Technau G M. Molecular markers for identified neuroblasts in the developing brain of Drosophila. Development, 2003, 130: 3621-3637

19 Schmucker D, Jackle H, Gaul U. Genetic analysis of the larval optic nerve projection in Drosophila. Development, 1997, 124: 937-948

20 Garcia-Alonso L, VanBerkum M F, Grenningloh G, et al. Fasciclin II controls proneural gene expression in Drosophila. Proc Natl Acad Sci USA, 1995, 92: 10501-10505

21 Vactor D V, Sink H, Fambrough D, et al. Genes that control neuromuscular specificity in Drosophila. Cell, 1993, 73: 1137-1153

22 Davis G W, Schuster C M, Goodman C S. Genetic analysis of the mechanisms controlling target selection: target-derived Fasciclin II regulates the pattern of synapse formation. Neuron, 1997, 19: 561-573

23 Lin D M, Fetter R D, Kopczynski C, et al. Genetic analysis of Fasciclin II in Drosophila: defasciculation, refasciculation, and altered fasciculation. Neuron, 1994, 13: 1055-1069

24 Lin D M, Goodman C S. Ectopic and increased expression of Fasciclin II alters motoneuron growth cone guidance. Neuron, 1994, 13: $507-523$

25 Sanchez-Soriano N, Prokop A. The influence of pioneer neurons on a growing motor nerve in Drosophila requires the neural cell adhesion molecule homolog FasciclinII. J Neurosci, 2005, 25: 78-87

26 Kohsaka H, Takasu E, Nose A. In vivo induction of postsynaptic molecular assembly by the cell adhesion molecule Fasciclin2. J Cell Biol, 2007, 179: 1289-1300

27 Morimoto T, Nobechi M, Komatsu A, et al. Subunit-specific and homeostatic regulation of glutamate receptor localization by CaMKII in Drosophila neuromuscular junctions. Neuroscience, 2010, 165: 1284-1292

28 Beumer $\mathrm{K}$, Matthies $\mathrm{H} \mathrm{J}$, Bradshaw A, et al. Integrins regulate DLG/FAS2 via a CaM kinase II-dependent pathway to mediate synapse elaboration and stabilization during postembryonic development. Development, 2002, 129: 3381-3391

29 Silies M, Klambt C. APC/C(Fzr/Cdh1)-dependent regulation of cell adhesion controls glial migration in the Drosophila PNS. Nat Neurosci, 2010, 13: 1357-1364

30 Woods D F, Hough C, Peel D, et al. Dlg protein is required for junction structure, cell polarity, and proliferation control in Drosophila epithelia. J Cell Biol, 1996, 134: 1469-1482

31 Chiba A, Snow P, Keshishian H, et al. Fasciclin III as a synaptic target recognition molecule in Drosophila. Nature, 1995, 374: 166-68

32 Alenius M, Bohm S. Identification of a novel neural cell adhesion molecule-related gene with a potential role in selective axonal projection. J Biol Chem, 1997, 272: 26083-26086 
33 Hortsch M. Structural and functional evolution of the L1 family: are four adhesion molecules better than one? Mol Cell Neurosci, 2000, 15: $1-10$

34 Yamamoto $\mathrm{M}$, Ueda $\mathrm{R}$, Takahashi $\mathrm{K}$, et al. Control of axonal sprouting and dendrite branching by the Nrg-Ank complex at the neuron-glia interface. Curr Biol, 2006, 16: 1678-1683

35 Hall S G, Bieber A J. Mutations in the Drosophila neuroglian cell adhesion molecule affect motor neuron pathfinding and peripheral nervous system patterning. J Neurobiol, 1997, 32: 325-340

36 Martin V, Mrkusich E, Steinel M C, et al. The L1-type cell adhesion molecule Neuroglian is necessary for maintenance of sensory axon advance in the Drosophila embryo. Neural Dev, 2008, 3: 10

37 Salinas P C, Price S R. Cadherins and catenins in synapse development. Curr Opin Neurobiol, 2005, 15: 73-80

38 Suzuki S C, Takeichi M. Cadherins in neuronal morphogenesis and function. Dev Growth Differ, 2008, 50 Suppl 1: S119-130

39 Yonekura S, Ting C Y, Neves G, et al. The variable transmembrane domain of Drosophila N-cadherin regulates adhesive activity. Mol Cell Biol, 2006, 26: 6598-6608

40 Iwai $\mathrm{Y}$, Usui $\mathrm{T}$, Hirano $\mathrm{S}$, et al. Axon patterning requires DN-cadherin, a novel neuronal adhesion receptor, in the Drosophila embryonic CNS. Neuron, 1997, 19: 77-89

41 Schmucker D, Clemens J C, Shu H, et al. Drosophila Dscam is an axon guidance receptor exhibiting extraordinary molecular diversity. Cell, 2000, 101: 671-684

42 Soba P, Zhu S, Emoto K, et al. Drosophila sensory neurons require Dscam for dendritic self-avoidance and proper dendritic field organization. Neuron, 2007, 54: 403-416

43 Hattori D, Chen Y, Matthews B J, et al. Robust discrimination between self and non-self neurites requires thousands of Dscam1 isoforms. Nature, 2009, 461: 644-648

44 Bhat M A, Rios J C, Lu Y, et al. Axon-glia interactions and the domain organization of myelinated axons requires neurexin IV/Caspr/Paranodin. Neuron, 2001, 30: 369-383

45 Baumgartner S, Littleton J T, Broadie K, et al. A Drosophila neurexin is required for septate junction and blood-nerve barrier formation and function. Cell, 1996, 87: 1059-1068

46 Bhat M A, Izaddoost S, Lu Y, et al. Discs Lost, a novel multi-PDZ domain protein, establishes and maintains epithelial polarity. Cell, 1999, 96: 833-845

47 Banerjee S, Blauth K, Peters K, et al. Drosophila neurexin IV interacts with roundabout and is required for repulsive midline axon guidance. J Neurosci, 2010, 30: 5653-5667

48 Wheeler S R, Banerjee S, Blauth K, et al. Neurexin IV and Wrapper interactions mediate Drosophila midline glial migration and axonal ensheathment. Development, 2009, 136: 1147-1157

49 Zweier C, de Jong E K, Zweier M, et al. CNTNAP2 and NRXN1 are mutated in autosomal-recessive Pitt-Hopkins-like mental retardation and determine the level of a common synaptic protein in Drosophila. Am J Hum Genet, 2009, 85: 655-666

50 Chen K, Gracheva E O, Yu S C, et al. Neurexin in embryonic Drosophila neuromuscular junctions. PLoS One, 2010, 5: e11115

51 Li J, Ashley J, Budnik V, et al. Crucial role of Drosophila neurexin in proper active zone apposition to postsynaptic densities, synaptic growth, and synaptic transmission. Neuron, 2007, 55: 741-755

52 Zeng X, Sun M, Liu L, et al. Neurexin-1 is required for synapse formation and larvae associative learning in Drosophila. FEBS Lett, 2007, 581: 2509-2516

53 Sun M, Xing G, Yuan L, et al. Neuroligin 2 is required for synapse development and function at the Drosophila neuromuscular junction. J Neurosci, 2011, 31: 687-699

54 Banovic D, Khorramshahi O, Owald D, et al. Drosophila neuroligin 1 promotes growth and postsynaptic differentiation at glutamatergic neuromuscular junctions. Neuron, 2010, 66: 724-738

55 Dean C. Neuroligins and neurexins: linking cell adhesion, synapse formation and cognitive function. Trends Neurosci, 2006, 29: 21-29. Epub 2005 Dec 2007

56 Sun M, Liu L, Zeng X, et al. Genetic interaction between Neurexin and $\mathrm{CAKI} / \mathrm{CMG}$ is important for synaptic function in Drosophila neuromuscular junction. Neurosci Res, 2009, 64: 362-371

57 Kittel R J, Wichmann C, Rasse T M, et al. Bruchpilot promotes active zone assembly, $\mathrm{Ca}^{2+}$ channel clustering, and vesicle release. Science, 2006, 312: 1051-1054

58 Wagh D A, Rasse T M, Asan E, et al. Bruchpilot, a protein with homology to ELKS/CAST, is required for structural integrity and function of synaptic active zones in Drosophila. Neuron, 2006, 49: 833-844

59 Owald D, Sigrist S J. Assembling the presynaptic active zone. Curr Opin Neurobiol, 2009, 19: 311-318

60 Owald D, Fouquet W, Schmidt M, et al. A Syd-1 homologue regulates pre- and postsynaptic maturation in Drosophila. J Cell Biol, 2010, 188: 565-579

61 de Wit J, Sylwestrak E, O'Sullivan M L, et al. LRRTM2 interacts with Neurexin 1 and regulates excitatory synapse formation. Neuron, 2009, 64: 799-806

62 Ko J, Fuccillo M V, Malenka R C, et al. LRRTM2 functions as a neurexin ligand in promoting excitatory synapse formation. Neuron, 2009, 64: 791-798

Open Access This article is distributed under the terms of the Creative Commons Attribution License which permits any use, distribution, and reproduction in any medium, provided the original author(s) and source are credited. 